\title{
Implementation of IoT in Agriculture
}

\author{
B.Mohamed Arafath Rajack a,1, N.Subramanian ${ }^{\text {a }}$, N. Arun Pragadesh ${ }^{\text {a }}$, R.Suvanesh ${ }^{\text {a }}$, \\ S.Vignesh ${ }^{\text {a }}$ \\ ${ }^{a}$ Department of Electrical and Electronics Engineering, SRM TRP Engineering \\ College, Trichy
}

\begin{abstract}
In this modern world agriculture is one of the major booming sectors around the world. In India around 60 percent of GDP comes from agriculture sector alone. Also, around the world there are many technologies showing up in the field of agriculture. In this paper proposed a technology by means of which potential pest attack in the crops can be detected and the respective pesticide is also sprayed as well. Along with these there is a range of sensor employed in the field connected to the controller that will take the real time values from the field and can be displayed in the respective screen (monitor or mobile screen) by means of technology called IOT (Internet of Things). Raspberry-pi is used as the controller to perform IoT. system is linked with an application called "cain" Which allows us to display various values of sensors in the monitor or in mobile application.
\end{abstract}

Keywords. Internet of Things, Raspberry-pi, Sensors

\section{Introduction}

Agriculture is one of the most important and booming sector around the world lots of new and advanced technology is been introduced in this field. It is quite vulnerable field too because due to a single sign of disease can destroy the entire crops in matter of days before the farmer can do anything. So, it becomes really important to identify the kind of pest attack and take action immediately. Here, image processing technology is used to detect the pest in the plants [1]. This is achieved by preprocessing the image that are already stored in the storage system with the real time image captured by the camera that are installed in the fields over the crop .Here, image processing technology is used to detect the pest in the plants[2]. This is achieved by preprocessing the image that are already stored in the storage system with the real time image captured by the camera that are installed in the fields over the crop.

\section{Implementation of IoT}

The Internet of Things (IoT) refers to a system of interrelated, internet-connected objects that are able to collect and transfer data over a wireless network without human intervention. The personal or business possibilities are endless [3]. The interconnection of these multiple embedded devices will be resulting in automation in nearly all fields and also enabling advanced applications. 
This is resulting in improved accuracy, efficiency, and economic benefit with reduced human intervention [4]. The IoT system is shown in Figure 1.

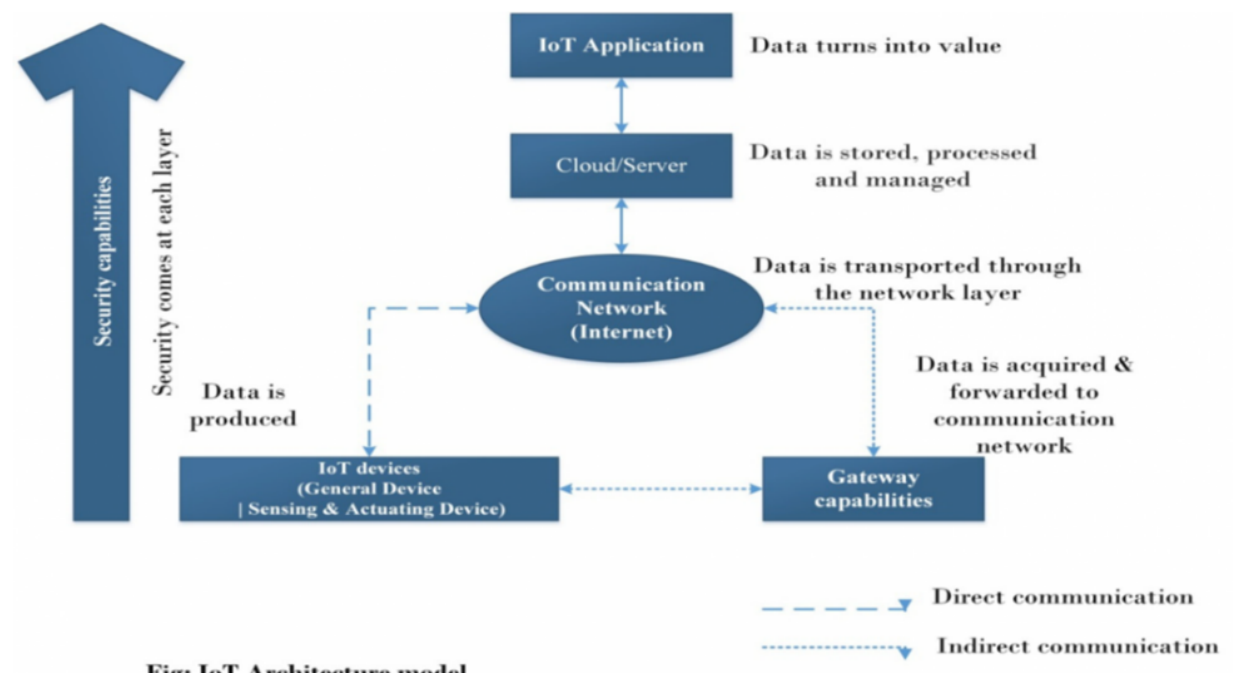

Fig: IoT Architecture model

Figure 1. Convolutional Neural Network (CNN)

\section{Convolution Neural Network (CNN)}

This is the main part of the system in which image processing is done. CNN is a Deep Learning algorithm method which can intake a image from a user and assign various weights to it by converting it into the array to various aspects and object so that the algorithm can differentiate one image from the other [5]. It is required to preprocess Conv-Net is much lower in comparison to other algorithm's classification. This algorithm is primary need for the applications involving image processing and we have also used the same in it [6-8].

\section{Block Diagram}

As shown in Figure 2, the controller is the central part of the system and there are various sensors like PH sensor, Soil moisture sensor, humidity sensor and all other kind of sensors as well which need to be deployed We can see a SD card module at the bottom of the micro controller. This is been used as the controller can't hold all the programs. So for some additional memory the SD card is been used to store the programs 
Power supply for all unit

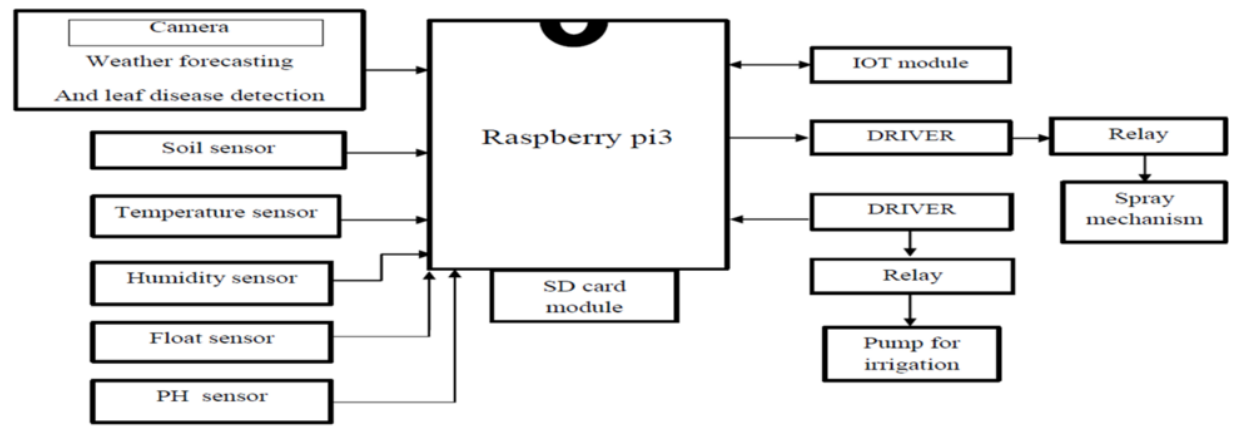

Figure 2. Hardware Block Diagram of Convolutional Neural Network (CNN)

\section{Conclusion}

A complete automatic crop disease detection system is proposed. The hardware components used to build the system with all their specification is discussed in the above section. Also, the software requirements for the system are also discussed in the above section. A prototype was designed with various type of sensors that will collect values from the soil sample collected from the fields and the result is also tested as well. Then the diseased plant is brought and tested with the algorithm we have in the system. This system can be further deployed in the practical field in the future and we can make it as a standalone system by installing a suitable solar panel.

\section{References}

[1]. S. D. Khirade and A. B. Patil, "Plant Disease Detection Using Image Processing," 2015 International Conference on Computing Communication Control and Automation, 2015, pp. 768-771, doi: 10.1109/ ICCUBEA.2015.153.

[2]. Shandilya, U., \& Khanduja, V. (2020). Intelligent farming system with weather forecast support and crop prediction. In Proceedings of the 2020 International Conference on Computing, Communication and Security, ICCCS 2020. Institute of Electrical and Electronics Engineers Inc. https://doi.org/10.1109/ICCCS49678.2020.9277437

[3]. Dahane, A., Benameur, R., Kechar, B., \& Benyamina, A. (2020). An IoT based smart farming system using machine learning. In 2020 International Symposium on Networks, Computers and Communications, ISNCC 2020. Institute of Electrical and Electronics Engineers Inc. https://doi.org/10.1109/ISNCC49221.2020.9297341

[4]. Marcu, I. M., Suciu, G., Balaceanu, C. M., \& Banaru, A. (2019). IoT based System for Smart Agriculture. In Proceedings of the 11th International Conference on Electronics, Computers and Artificial Intelligence, ECAI 2019. 
Institute of Electrical and Electronics Engineers Inc. https://doi.org/10.1109/ECAI46879.2019.9041952

[5]. Singh, R., Srivastava, S., \& Mishra, R. (2020). AI and IoT Based Monitoring System for Increasing the Yield in Crop Production. In International Conference on Electrical and Electronics Engineering, ICE3 2020 (pp. 301305). Institute of Electrical and Electronics Engineers Inc. https://doi.org/10.1109/ICE348803.2020.9122894

[6]. Ayaz, M., Ammad-Uddin, M., Sharif, Z., Mansour, A., \& Aggoune, E. H. M. (2019). Internet-of-Things (IoT)-based smart agriculture: Toward making the fields talk. IEEE Access, 7, 129551-129583. https://doi.org/10.1109/ ACCESS.2019.2932609

[7]. Ganesh Babu R., Elangovan K., Maurya S., Karthika P. (2021) Multimedia Security and Privacy on Real-Time Behavioral Monitoring in Machine Learning IoT Application Using Big Data Analytics. In: Kumar R., Sharma R., Pattnaik P.K. (eds) Multimedia Technologies in the Internet of Things Environment. Studies in Big Data, vol 79. Springer, Singapore. https://doi.org/10.1007/978-981-15-7965-3_9

[8]. Vengatesan, K., Kumar, A., Parthibhan, M., Singhal, A., \& Rajesh, R. (2018, December). Analysis of Mirai Botnet Malware Issues and Its Prediction Methods in Internet of Things. In International conference on Computer Networks, Big data and IoT (pp. 120-126).

[9]. Markkandan, S., Narayanan, L., Robert Theivadas, J., \& Suresh, P. (2021). Edge based interpolation with refinement algorithm using EDGE strength filter for digital camera images. Turkish Journal of Physiotherapy and Rehabilitation, 32(2), 981-993 